\title{
Osmotic Responsiveness and Cross Talk Involving Oxytocin, but Not Vasopressin or Amino Acids, between the Supraoptic Nuclei in Virgin and Lactating Rats
}

\author{
Inga Neumann, ${ }^{1}$ Rainer Landgraf, ${ }^{1}$ Lorenzo Bauce, ${ }^{2}$ and Quentin J. Pittman ${ }^{2}$ \\ ${ }^{1}$ Max Planck Institute of Psychiatry, Clinical Institute, 80804 Munich, Germany, and ${ }^{2}$ Neuroscience Research Group \\ and Department of Medical Physiology, University of Calgary, Calgary, Alberta, Canada
}

Lactation is associated with complex changes of the hypothalamo-neurohypophysial system, and oxytocin released within the hypothalamic supraoptic (SON) and paraventricular nuclei may serve as a signal of communication between the magnocellular nuclei in lactating rats. In the first study, the intranuclear and peripheral release patterns of oxytocin and vasopressin in response to intraperitoneal hypertonic saline were studied in virgin and lactating rats to determine if the reduced osmoresponsiveness of the oxytocinergic and vasopressinergic systems during lactation is reflected by reduced release not only into blood, but also within the SON. Simultaneous microdialysis was performed within the SON and the jugular vein before and up to $6 \mathrm{hr}$ after peripheral osmotic stimulation $(3.0 \mathrm{M} \mathrm{NaCl}, 0.6$ $\mathrm{ml} / 100 \mathrm{gm}$ body weight, i.p.). There was an immediate increase in secretion of both oxytocin and vasopressin into blood, whereas peptide release within the SON was delayed and peaked after 4-5 hr. Peripheral release of both peptides was significently reduced in lactating animals, whereas within the SON release of oxytocin, but not vasopressin, was significantly reduced during lactation.

In the second study, cross talk between the SONs-another phenomenon which seems to be characteristic for lactation-was studied. Microdialysis of one SON with hypertonic perfusion medium (with $1 \mathrm{~m} \mathrm{NaCl}$ ) significantly increased the release of oxytocin, vasopressin, and various amino acids (aspartate, glutamate, serine, glutamine, $\gamma$ amino butyric acid, and arginine) within the ipsilateral SON. In contrast to virgin female and male animals, this unilateral stimulation of the SON resulted in a transiently increased release of oxytocin in the contralateral SON of lactating rats. The release of vasopressin and amino acids within the contralateral SON of lactating rats remained unchanged, indicating specific activation of contralateral oxytocinergic neurons.

[Key words: cross talk, m/crodlalys/s, lactation, oxytocin vasopressin, supraoptic nucleus, osmoresponse, amino acids]

\footnotetext{
Received Aug. 19, 1994; accepted Nov. 9, 1994

We are grateful to Dr. J. Summy-Long (Hershey, PA) for critical reading of the manuscript and to Y. Takahashi for technical support. This work was supported by HFSOP (Strasbourg; I.N.) and AHFMR (I.N.) as well as MRC, VW, and NATO. Q.J.P. is an AHFMR scientist.

Correspondence should be addressed to Dr. Inga Neumann, Max Planck Institute of Psychiatry, Clinical Institute, Krepelinstrasse 2, 80804 Munich, Germany.

Copyright $\odot 1995$ Society for Neuroscience $0270-6474 / 95 / 153408-10 \$ 05.00 / 0$
}

Lactation is associated with complex morphological (Theodosis et al., 1986a) and physiological changes of the hypothalamoneurohypophysial system. In lactating rats, a blunted release of oxytocin (OXT) into blood was demonstrated in response to immobilization stress (Carter and Lightman, 1987; Higuchi et al., 1988), intravenous CCK-8 (Iliguchi et al., 1991), and CRH (Patel et al., 1991). Furthermore, despite an increased fluid loss due to nursing, the osmotic responsiveness of the oxytocinergic system becomes attenuated, as demonstrated by reduced release of OXT from the neurohypophysis into blood after systemic osmotic stimulation (Hartman et al., 1987; Higuchi et al., 1988; Koehler et al., 1993).

In addition to its release from neurohypophysial terminals, OXT is also released within the SON itself, for example, during suckling (Moos et al., 1989; Neumann et al., 1993a), parturition (Neumann et al., 1993a), or in response to osmotic stimulation of the nucleus by microdialysis of hypertonic medium (Neumann et al., 1993a,b). Intraperitoneal administration of hypertonic saline was shown recently to result in a small and delayed (compared to release into the blood stream) release of OXT within the SON in male rats (Neumann et al., 1994). Such locally released OXT may originate from dendrites and/or cell bodies as demonstrated histologically (Pow and Morris, 1989). In the present studies we examined the release of OXT and arginine vasopressin (AVP) within both the SON and into blood in response to intraperitoneal hypertonic saline over a $7 \mathrm{hr}$ period, and compared virgin female and lactating rats to determine if the alteration in peripheral release patterns during lactation is also accompanied by changes in the intranuclear release of both OXT and AVP within the SON. The use of microdialysis performed simultancously within the SON and the jugular vein enabled us to monitor and to compare intranuclear and peripheral peptide release.

In addition to intranuclear communication among OXT neurons (Theodosis, 1985), there is also evidence for communication among the various hypothalamic OXT-containing nuclei in the lactating rat. This is indicated by a periodic bursting activity of OXT neurons which is synchronized among the paired SON and paraventricular nuclei in response to suckling (Belin et al., 1984; Belin and Moos, 1986). Although connections between the SON and paraventricular nuclei are well established (Silverman et al., 1981; Saphier and Feldman, 1985, 1987), there is only limited anatomical evidence for connections between both SONs (Wakerley and Ingram, 1993). Indications for coordination between the SONs in lactating rats, however, are given by electrophysiological studies (Moos and Richard, 1989; Takano 
et al., 1990), and the question arises as to the ncurochemical signal(s) involved in the communication between the left and right SON. OXT itself seems to be a possible candidate for the following reasons. (1) OXT infused into the SON facilitates the occurrence and increases the amplitude of bursts in OXT cells in the contralateral SON (Moos and Richard, 1989). (2) Infusion of an OXT antagonist into the nucleus of lactating rats demonstrated that intranuclear OXT is involved in the further positive feedback activation of local OXT neurons (Neumann et al., 1994) and in the synchronization of the activity of OXT neurons located within different nuclei (Lambert et al., 1993). (3) OXT is released within the SON during local osmotic stimulation when glucose metabolism is enhanced on the contralateral SON (Summy-Long et al., 1994). Therefore, in the second part of these studies, we examined if stimulation of one SON by microdialysis of hypertonic medium induces OXT release not only within the ipsilateral (Neumann et al., 1993a,b) but also in the contralateral SON. By comparing the release of OXT within the contralateral SON of virgin female, lactating, and male rats, we asked if a possible alteration in the cross talk with OXT could account for the differences in responses between the different hormonal states. In addition, the release of AVP as well as candidate neurotransmitter amino acids, like glutamate, aspartate, $\gamma$ amino butyric acid (GABA), serine, and glutamine, known to be released by osmotic stimulation (Horn et al., 1992) was examined in the contralateral nucleus of lactating animals to answer the question as to the specificity of OXT released within the SON.

\section{Materials and Methods}

\section{Animals and surgery}

Experiments were performed on virgin female $(245-310 \mathrm{gm}$ body weight), lactating (days $7-10$ of lactation; $290-360 \mathrm{gm}$ body weight), and male (260-320 gm body weight) Sprague-Dawley rats (Charles River) housed under controlled laboratory conditions with free access to water and pelleted food. Litters of lactating rats were adjusted to $10-$ 12 pups postpartum.

\section{Surgery}

All animals were anesthetized with urethane (ethyl carbamate, $1.6 \mathrm{gm} /$ $\mathrm{kg}$, i.p.) throughout the entire experiment. Absence of withdrawal to toe pinch was verified throughout the experiment; and when necessary supplementary anesthetic was given. The body temperature was measured with a thermistor in the colon and maintained at $37^{\circ} \mathrm{C}$ with an electric heating pad.

In study 1 , the release of both OXT and AVP within the SON and from the neurohypophysis into blood was simultaneously monitored in response to intraperitoneal osmotic stimulation in virgin and lactating rats. Therefore, a U-shaped microdialysis probe (exposed membrane, $1.5 \mathrm{~mm}$; molecular cutoff, $6 \mathrm{kDa}$; in vitro relative recovery of OXT and AVP, between 1.7 and $2.0 \%$ ) was stereotaxically placed into the right SON (1.2 mm behind bregma, $1.8 \mathrm{~mm}$ lateral from midline, and 9.2 $\mathrm{mm}$ beneath the surface of the skull; Paxinos and Watson, 1986) and secured in place by stainless steel screws and dental cement. A second microdialysis probe (exposed membrane, $30 \mathrm{~mm}$; molecular cutoff, 50 $\mathrm{kDa}$; in vivo relative recovery of OXT, $60 \%$ ) was inserted into the jugular vein as described recently (Neumann et al., 1993b).

In study 2, the release of OXT within the left and right SON was studied in response to local osmotic stimulation of one SON in virgin female, lactating, and male rats; the release of AVP and amino acids was measured in lactating rats only. Therefore, a microdialysis probe was stereotaxically placed with its U-shaped tip into each SON. For simultaneous monitoring of OXT release into blood, a probe was placed into the right jugular vein as described above.

Immediately before implantation, the brain probes were filled with filtered $(0.22 \mu \mathrm{m}$, Millipore) artificial CSF (aCSF; composition, in mM: $\mathrm{NaCl}, 140 ; \mathrm{KCl}, 3.36 ; \mathrm{NaHCO}_{3}, 9.52 ; \mathrm{Na}_{2} \mathrm{HPO}_{4}, 0.49$; urea, 2.16; $\left.\mathrm{CaCl}_{2}, 1.26 ; \mathrm{MgCl}_{2}, 1.18 ; \mathrm{pH} 7.4\right)$ and the blood probes with sterile, heparinized saline $(100 \mathrm{IU} / \mathrm{ml})$. After completion of surgery, microdialysis probes were perfused with either aCSF (SON, for OXT and AVP detection), lactated Ringer's solution (SON; for amino acid detection; composition, in mM: $\mathrm{NaCl}, 130 ; \mathrm{KCl}, 4.0 ; \mathrm{CaCl}_{2}, 1.5 ;$ lactatc, $28.0 ; \mathrm{pH}$ 6.5 ), or heparinized saline (blood; $50 \mathrm{IU} / \mathrm{ml}$ ) for at least $60 \mathrm{~min}$ without sampling before the first basal samples (see below) were obtained in both studies 1 and 2. Our previous studies (Neumann et al., 1993b) indicate that peptide levels are stable at this time. All microdialysis probes were perfused at $3.8 \mu \mathrm{l} / \mathrm{min}$ throughout the experiments.

\section{Experimental protocol}

\section{Study 1: intraperitoneal hypertonic saline in virgin and} lactating rats

To investigate if the intranuclear and peripheral release of OXT and AVP in response to intraperitoneal hypertonic saline differs between virgin female and lactating, urethane-anesthetized rats, 15 consecutive 30 min microdialysates were sampled simultaneously from the microdialysis probe inserted with its tip into the right SON and from the probe inserted into the jugular vein of virgin (OXT, $n=10$; AVP, $n=$ 7) and lactating rats (OXT, $n=9$; AVP, $n=7$ ). Before starting the third collection period, hypertonic saline $(3 \mathrm{M} \mathrm{NaCl}, 0.6 \mathrm{ml} / 100 \mathrm{gm}$ body weight) was applied intraperitoneally.

\section{Study 2: cross talk between the left and right SON}

$O X T$ and $\Lambda V P$. In order to study the effect of local osmotic stimulation of one SON on the release of OXT within the ipsi- and contralateral SON in virgin female (OXT, $n=9$ ), lactating (OXT, $n=9$; AVP, $n=$ 6), and male urethane-anesthetized rats (OXT, $n=13$ ), 11 consecutive 30 min microdialysates were sampled simultaneously from the left and right SON and blood. During the third perfusion period, hypertonic aCSF (with $1 \mathrm{M} \mathrm{NaCl}$ ) instead of isotonic aCSF (with $0.14 \mathrm{M} \mathrm{NaCl}$ ) was perfused through the microdialysis probe located within the right SON. During the ninth perfusion period, hypertonic aCSF was perfused through the probe located within the left SON.

Amino acids. To monitor the release of glutamate, aspartate, serine, GABA, arginine, and glutamine within both SON of lactating rats $(n=$ 6 ) in response to local osmotic stimulation of one SON, six consecutive 15 min dialysates were sampled from the left and right SON. Hypertonic (with $1 \mathrm{M} \mathrm{NaCl}$ ) instead of isotonic Ringer solution was perfused through the left probe during the third perfusion period.

\section{Histology}

At the end of the experiment, the rats were killed by decapitation. Brains were removed and fixed in $10 \%$ formaldehyde. Frontal $40-60 \mu \mathrm{m}$ frozen sections were cut to verify the placement of the microdialysis probe histologically (Figs. 1, 2).

\section{Analysis of OXT and AVP by radioimmunoassay}

All microdialysates were stored at $-20^{\circ} \mathrm{C}$ and lyophilized until further assay. OXT and AVP content in microdialysates from the SON and blood were assayed using sensitive $(0.1 \mathrm{pg} / \mathrm{sample}$, both peptides) and specific (cross-reactivity with AVP and OXT, respectively, $<0.7 \%$ ) radioimmunoassays without extraction (Neumann et al., 1993a). All brain samples from one experiment (except microdialysates sampled from the SON of male rats in study 2), and all blood samples, were measured in the same assay to eliminate interassay variation.

\section{Analysis of amino acids}

For amino acid analysis, a $25 \mu \mathrm{l}$ aliquot of the dialysate was automatically derivatized with $50 \mu \mathrm{l}$ orthophthaldialdehyde (OPA) reagent (20 $\mathrm{mm}$ OPA, $2 \mu \mathrm{l} / \mathrm{ml} \beta$-mercaptoethanol, $1 \mu \mathrm{l} / \mathrm{ml}$ BRIJ 30 in $1 \mathrm{M}$ sodium borate buffer, $\mathrm{pH} \mathrm{10.4)} \mathrm{for} 1$ min using a Waters WISP 715 autosampler and separated at $35^{\circ} \mathrm{C}$ on a Beckman Ultrasphere $\mathrm{C}-18$ column $(4.5 \times$ $150 \mathrm{~mm}, 5 \mu \mathrm{m}$ ) using a mobile phase flowing $1.5 \mathrm{ml} / \mathrm{min}$ of $0.025 \mathrm{M}$ imidazole phosphate ( $\mathrm{pH} 6.5$ ), 5\% methanol, and $1.5 \%$ tetrahydrofuran, and a linear gradient of 0-50\% methanol. Detection was with a Varian Fluorichrom fluorimeter with tungsten/halogen lamp, $380 \mathrm{~nm}$ excitation filter, and $>450 \mathrm{~nm}$ emission filter. Quantitation was by means of peak area and the external standard method using Waters Maxima data acquisition and analysis software. The detection limit ( 10 times detector noise) was $1 \mathrm{pmol}$ of aspartate, $1.2 \mathrm{pmol}$ of glutamate, $0.6 \mathrm{pmol}$ of serine, $0.6 \mathrm{pmol}$ of glutamine, $1.0 \mathrm{pmol}$ of arginine, and $0.6 \mathrm{pmol}$ of GABA injected onto the column. 

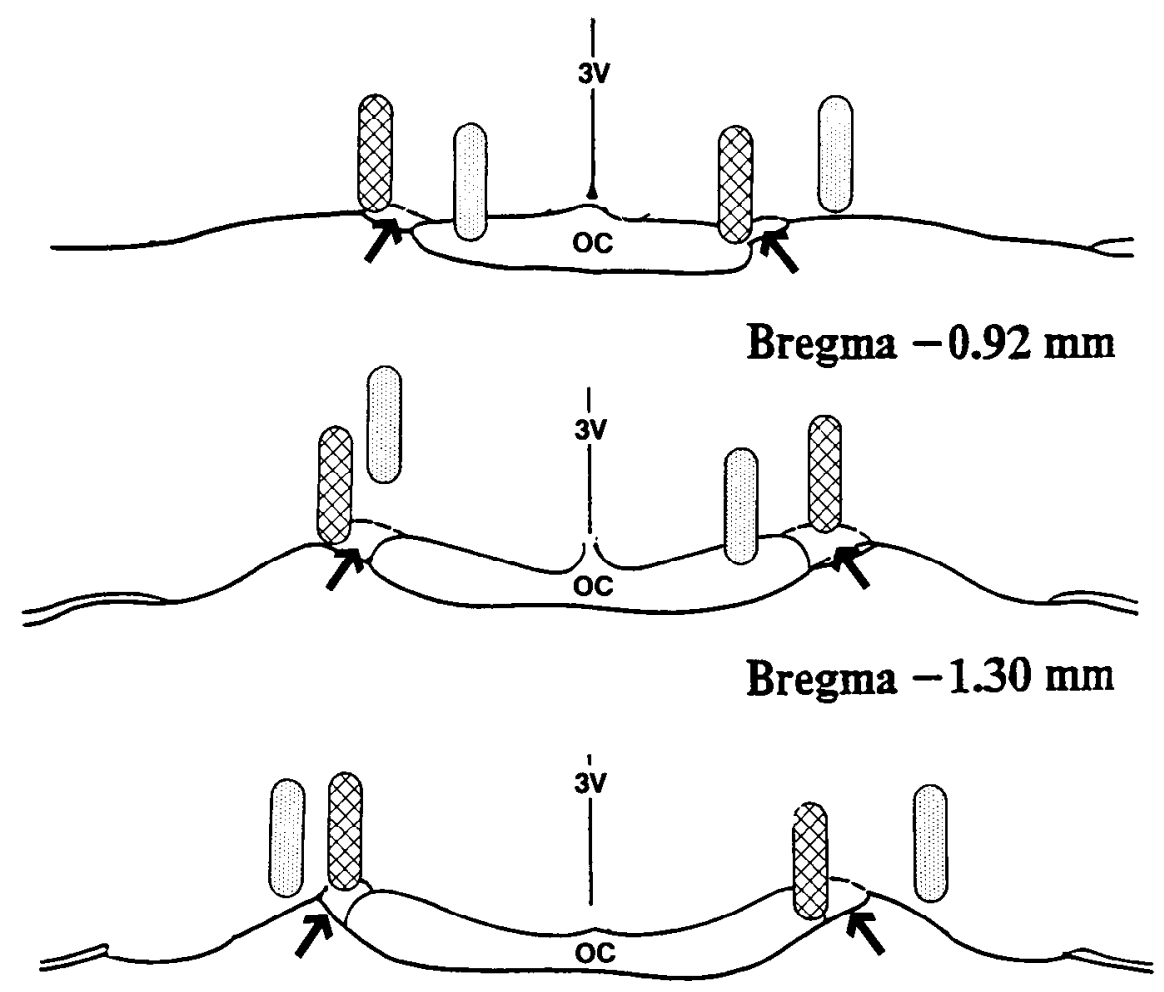

Figure 1. Typical sites of the microdialysis probe located within/adjacent to the hypothalamic supraoptic nucleus (SON) which were considered as a "hit" (hatched) and outside the SON which were considered as a "miss" (shaded). Arrows point to the SON. $3 \mathrm{~V}$, third cerebral ventricle; $O C$, optic chiasm: $O T$, optic tract. Adapted from Paxinos and Watson (1986).

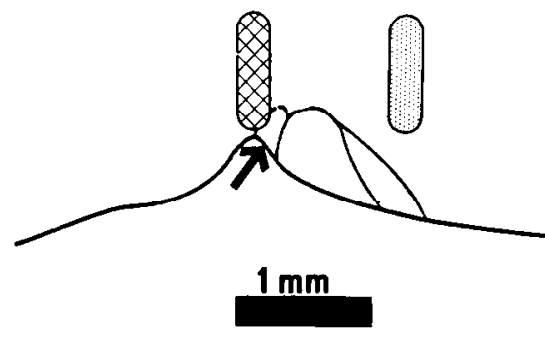

\section{Bregma $-1.40 \mathrm{~mm}$}

\section{Statistics}

All data are expressed as the mean \pm SEM. Two-way analysis of variance for repeated measurements followed by Dunnett's test or by Bonferroni $t$ test to determine differences within groups and by NewmanKeuls test to determine differences between virgin and lactating animals were used as indicated (SIGMASTAT). One-way analysis of variance for repeated measurements followed by Dunnett's test was used for statistical analysis of OXT content in dialysates sampled from the left and right SON of male rats (study 2 ). $P<0.05$ was considered statistically significant.

\section{Results}

Experimental data from a particular animal were used only if microdialysis probes were placed within the right SON (study 1) or within both the left and right SON (study 2). A microdialysis placement was considered a "hit" if (1) its placement could be histologically verified to be in or immediately adjacent to the SON (see Figs. 1, 2) and (2) direct osmotic stimulation of the perfused area resulted in an increase in OXT content in the dialysate.

\section{Study 1: intraperitoneal hypertonic saline in virgin and lactating rats}

Peripheral osmotic stimulation resulted in a delayed but significant rise in intranuclear OXT release which did not occur before the second perfusion period following administration of intra- peritoneal hypertonic saline and which continued until $6 \mathrm{hr}$ after stimulation in virgin and lactating rats $(p<0.001$; Fig. 3). Whereas basal release within the SON did not differ significantly between virgin and lactating rats, OXT release within the SON in lactating rats after stimulation was only about one-third that of virgin rats $(p<0.023$; Fig. 3 ).

Sinultaneously sampled dialysates from blood revealed an immediate rise in OXT secretion into blood in both groups ( $p$ $<0.001)$. Although prestimulation levels differed significantly $(p<0.05)$ between virgin $(1.12 \pm 0.22 \mathrm{pg} /$ dialysate $)$ and lactating animals $(0.37 \pm 0.04 \mathrm{pg} /$ dialysate $), \mathrm{OXT}$ content in dialysates increased 12 -fold in both groups in the poststimulation period. Thus, absolute OXT content in dialysates sampled from blood after intraperitoneal hypertonic saline differed significantly over the $6 \mathrm{hr}$ poststimulation sampling period between both groups ( $p<0.001$; Fig. 3 ).

Similar to OXT, the rise in the release of AVP within the SON after intraperitoneal hypertonic saline was delayed and did not occur before starting the second perfusion period after osmotic stimulation in both groups $(p<0.001$; Fig. 4$)$. There was no difference in basal or stimulated release of AVP within the SON between virgin and lactating rats (Fig. 4). In contrast, the immediate rise in AVP secretion into blood was higher in virgin (fourfold) than in lactating animals (2.8-fold, $p<0.05$; Fig. 4). 

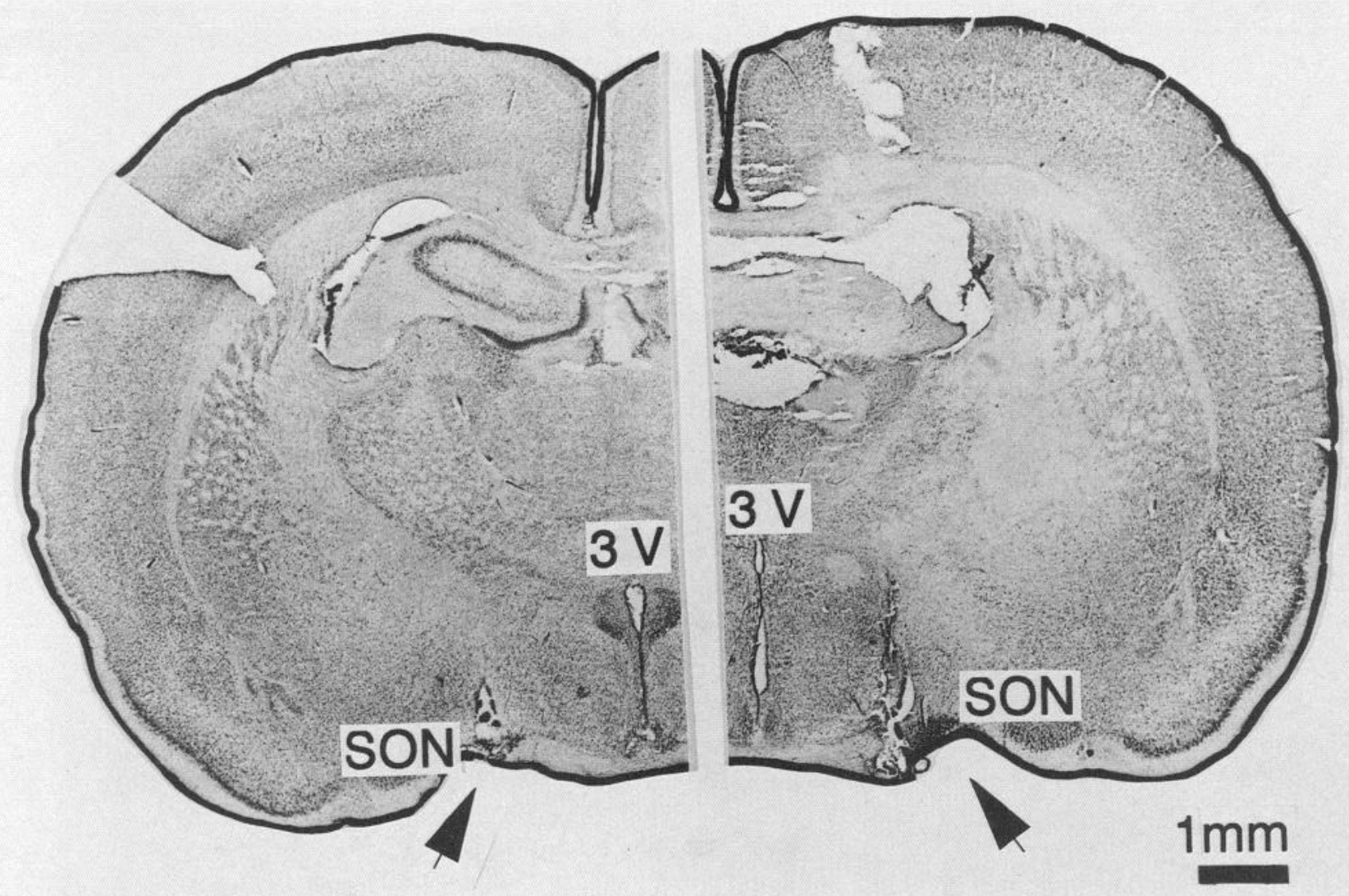

Figure 2. Two representative frontal sections through the brain demonstrating the microdialyzed SON area after removal of the probe. $3 \mathrm{~V}$, third ventricle; SON, supraoptic nucleus.

\section{Study 2: cross talk between the left and right SON}

$O X T$. Basal content of OXT was detectable in microdialysates sampled from both the left and right SON of virgin female (left, $0.59 \pm 0.13 \mathrm{pg}$; right, $0.93 \pm 0.30 \mathrm{pg})$, lactating $(0.41 \pm 0.10$ $\mathrm{pg}, 0.82 \pm 0.22 \mathrm{pg})$, and male rats $(1.09 \pm 0.32 \mathrm{pg}, 1.04 \pm$ $0.22 \mathrm{pg}$ ). During perfusion of hypertonic aCSF through the microdialysis probe located in the right SON, OXT content in ipsilateral (right) dialysates was increased 12-fold (virgin, $p<$ 0.001 ), 4.5-fold (lactating, $p<0.05$ ), and 9-fold (male, $p<$ $0.001)$. In lactating rats, the increase in intranuclear OXT release in response to local osmotic stimulation was significantly less $(p<0.05)$ than in virgin rats (Fig. 5). In microdialysates sampled simultaneously from the contralateral (left) SON, OXT content tended to increase 1.4 -fold in both virgin female and lactating rats (Fig. 5), whereas it was virtually unchanged in male rats (Fig. 6). When the hypertonic perfusion medium was replaced in the right SON by isotonic aCSF again, OXT release in the right SON remained high in virgin, lactating, and male rats (Figs. 5, 6), as described before. In the contralateral SON, the increase in OXT release reached statistical significance in lactating rats ( $p<0.05$ vs prestimulation; Fig. 5), but not in virgin (Fig. 5) and male rats (Fig. 6). Three hours after osmotic stimulation of the right SON, osmotic stimulation of the left SON resulted in significant increase in the release of OXT within the left SON in all three groups (Figs. 5, 6). In simultaneously sampled dialysates from the contralateral (right) SON, OXT contents tended to increase in both lactating (1.6-fold) and virgin (1.5-fold) rats compared to the immediate prestimulation levels, but this increase did not reach statistical significance. Again, during direct osmotic stimulation of the left SON in male rats, OXT release in the contralateral SON remained unchanged (Fig. 6).

In two lactating and two virgin female rats, one microdialysis probe was placed outside $(300-500 \mu \mathrm{m}$ lateral or caudomedial) the SON. OXT content in dialysates sampled from those probes was found to be only slightly increased during ipsilateral (lactating rats, from 0.34 to $0.56 \mathrm{pg}$; virgin rats, from 0.42 to 1.17 pg) and even slightly decreased during contralateral perfusion with hypertonic aCSF (from 0.39 to $0.25 \mathrm{pg}$ and from 0.35 to $0.25 \mathrm{pg}$, respectively).

In microdialysates sampled within the jugular vein, detectable levels of OXT were found under basal conditions in virgin female $(0.50 \pm 0.14 \mathrm{pg} /$ dialysate $)$ and lactating $(0.29 \pm 0.07 \mathrm{pg} /$ dialysate) rats. During sequential unilateral hypertonic aCSF microdialysis of the left/right SON, OXT content in dialysates from blood was found to be increased in both virgin and lactating rats. In lactating animals, however, OXT release into blood was significantly less $(p<0.05)$ than in virgin rats (Fig. 5) regardless of whether the right or left SON was osmotically stimulated via the microdialysis probe.

$A V P$. In a follow-up study, basal (prestimulation) levels of AVP did not differ in $30 \mathrm{~min}$ microdialysates sampled from the left and right SON of lactating rats. In response to perfusion of hypertonic aCSF through the left probe, a significant increase (up to sixfold, $p<0.05$ ) in ipsilateral AVP release was found as expected, whereas release in the contralateral SON remained unchanged (Table 1). 


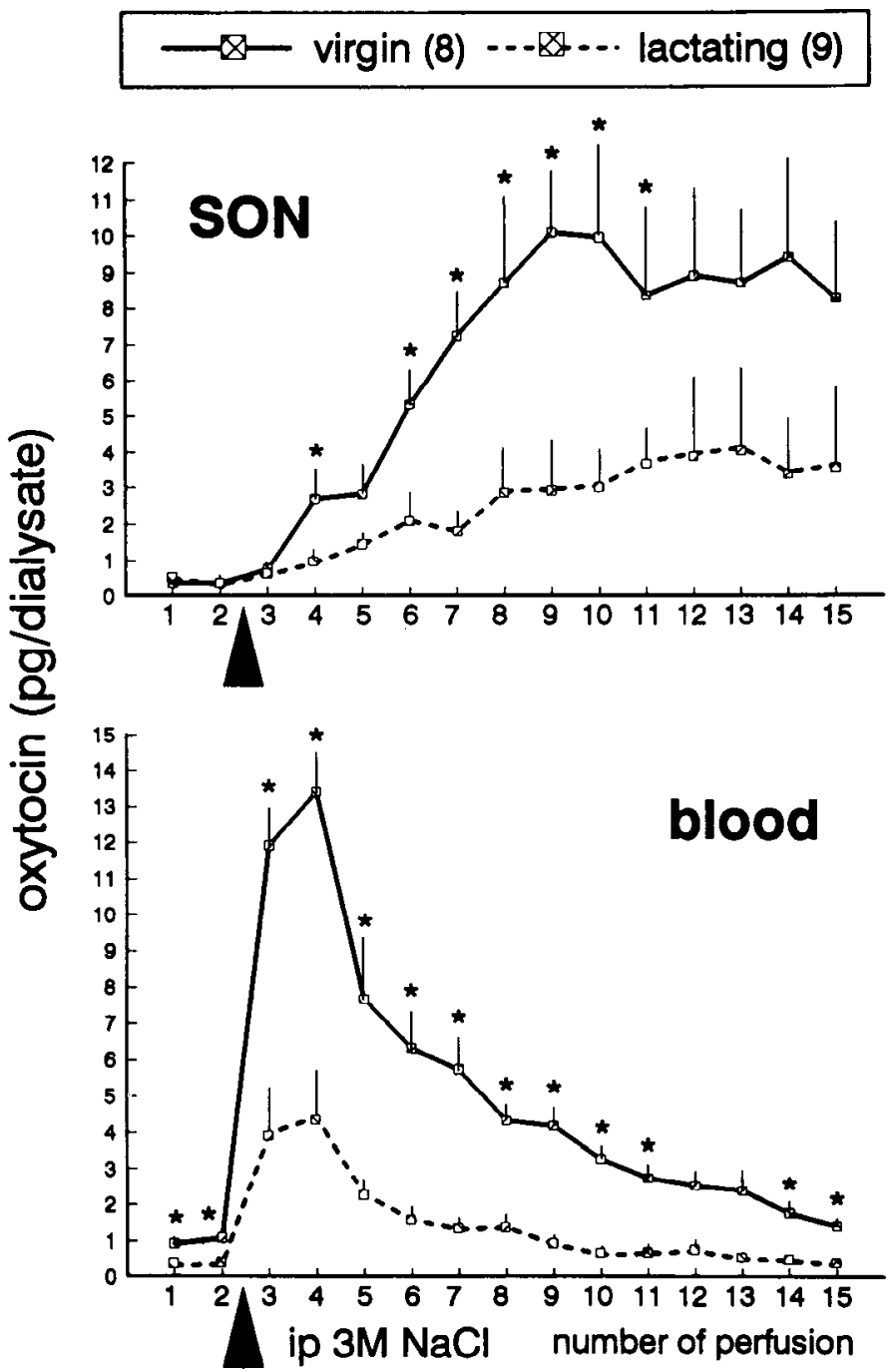

Figure 3. OXT content in 15 consecutive 30 min dialysates sampled from the SON and the jugular vein of virgin and lactating urcthancanesthetized rats. Before starting the third perfusion, hypertonic saline $(3.0 \mathrm{M}, 0.6 \mathrm{ml} / 100 \mathrm{gm}$ body weight, i.p.) was administered. Numbers in parentheses indicate group size. Values are the mean \pm SEM. Twoway ANOVA for repeated measurements revealed statistically significant differences due to treatment and group: $p<0.023$ (SON), $p<$ 0.001 (blood). $*, p<0.05$ versus corresponding values from lactating animals (Newman-Keuls' test).

Amino acids. Basal levels of aspartate $(6.33 \pm 1.33 \mathrm{pmol})$, glutamate $(10.5 \pm 2.32 \mathrm{pmol})$, serine $(38.3 \pm 6.67 \mathrm{pmol})$, glutamine $(331 \pm 33.2$ pmol $)$, GABA $(10.8 \pm 2.35 \mathrm{pmol})$, and arginine $(20.6 \pm 4.32 \mathrm{pmol})$ were detectable in 10 min microdialysates sampled from the left SON of lactating rats and did not significantly differ from those sampled from the right SON. During perfusion of hypertonic aCSF through the left probe, a significant increase in the release of all amino acids estimated was found in the left SON (Figs. 7, 8). After replacement of hypertonic with isotonic medium, the release of all of the amino acids monitored in the left SON tended to decrease, but was still significantly higher than during the prestimulation period (Fig. 7). In contrast to the stimulated site, there was no increase in any amino acid release in the contralateral SON during or after perfusion with hypertonic medium.
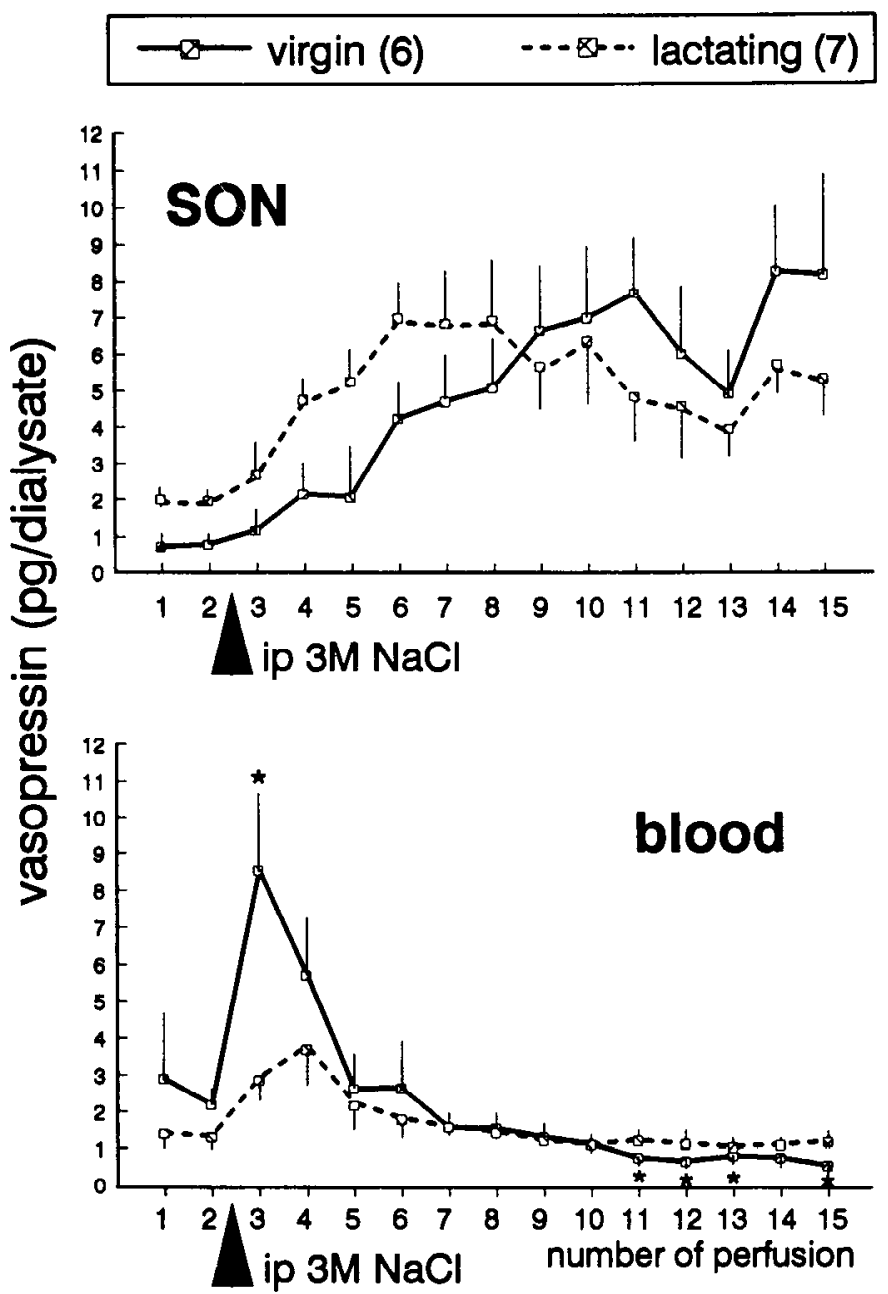

Figure 4. AVP content in dialysates sampled from the SON and blood of virgin female and lactating urethane-anesthetized rats. For other details see Figure 3 legend. Two-way ANOVA for repeated measurements revealed significant differences due to treatment and group $(p<0.001$, blood) or due only to treatment $\left(p<0.001\right.$, SON). ${ }^{*}, p<0.05$ versus corresponding value from lactating animals (Newman-Keuls' test).

\section{Discussion}

The results of this study provide three major new findings. (1) The reduced osmoresponsiveness of the oxytocinergic and vasopressinergic systems in lactating rats is reflected not only by a reduced release into blood, but also by reduced OXT release within the hypothalamic SON after systemic (intraperitoneal) osmotic stimulation. Independent of lactation, the release of both neuropeptides within the SON is delayed compared to release patterns into blood, indicating independent regulatory mechanisms. (2) Osmotic stimulation of one SON significantly stimulates the release of OXT within the contralateral SON in lactating but not virgin female and male rats. (3) The direct osmotic stimulation of one SON specifically activates contralateral OXT neurons in lactating rat as the release of AVP and various candidate transmitter amino acids within the contralateral SON remained unchanged.

Response to intraperitoneal hypertonic saline in virgin and lactating rats

A reduced sensitivity of the oxytocinergic system to a variety of stimuli such as hyperosmolality of plasma (Hartman et al., 

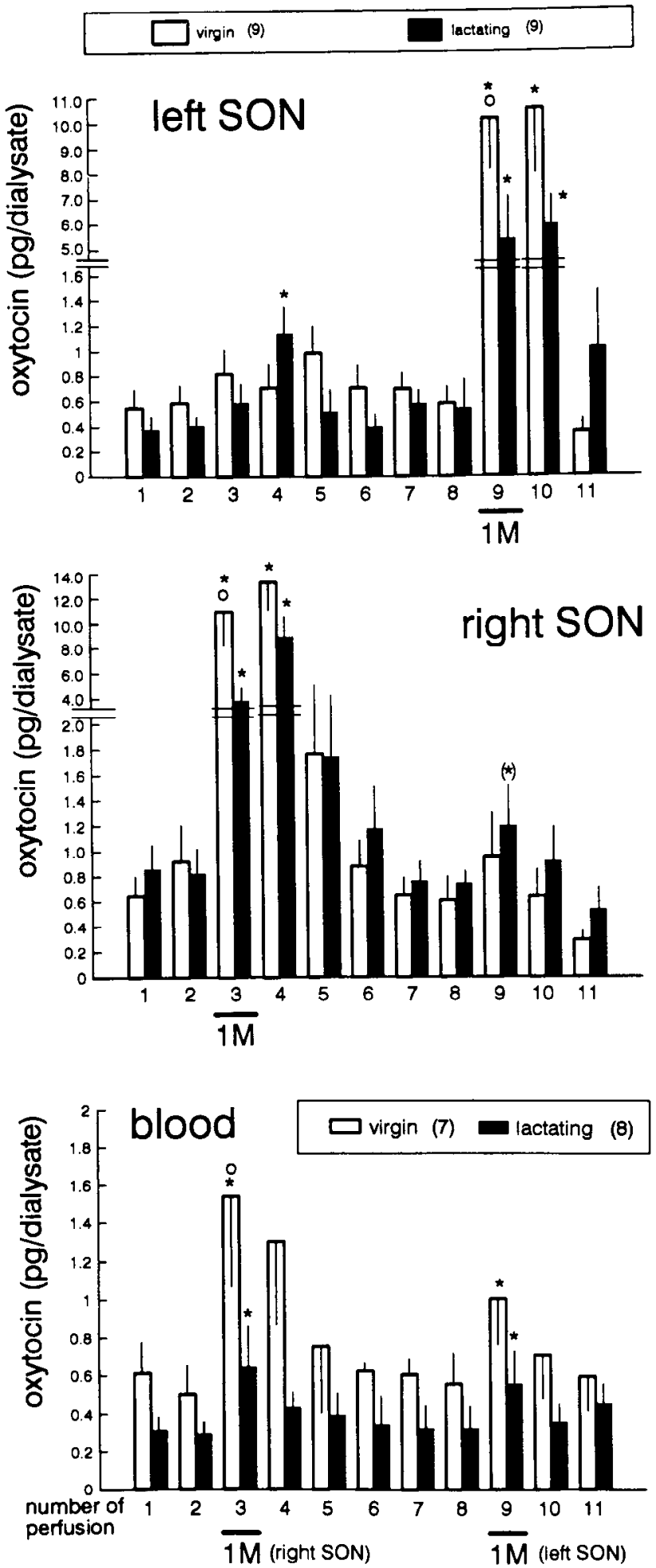

Figure 5. OXT content in 11 consecutive 30 min dialysates sampled simultaneously within the left (top) and right SON (middle) as well as from the jugular vein (bottom) of virgin female and lactating urethane-anesthetized rats. Hypertonic aCSF (with $1 \mathrm{M} \mathrm{NaCl}$ ) instead of isotonic aCSF was perfused through the right dialysis probe during the third and through the left dialysis probe during the ninth perfusion period. Number in parentheses indicates group size. Values are the mean \pm SEM. Two-way ANOVA for repeated measurements: $p<$ 0.001 (both left and right SON); $p<0.01$ (blood) followed by Dunnett's $t$ test, $*, p<0.05$ versus respective prestimulation, or NewmanKeuls' test, $\circlearrowleft, p<0.05$ versus lactating. ${ }^{*}, p<0.05$ paired $t$ test versus perfusion number 8 .

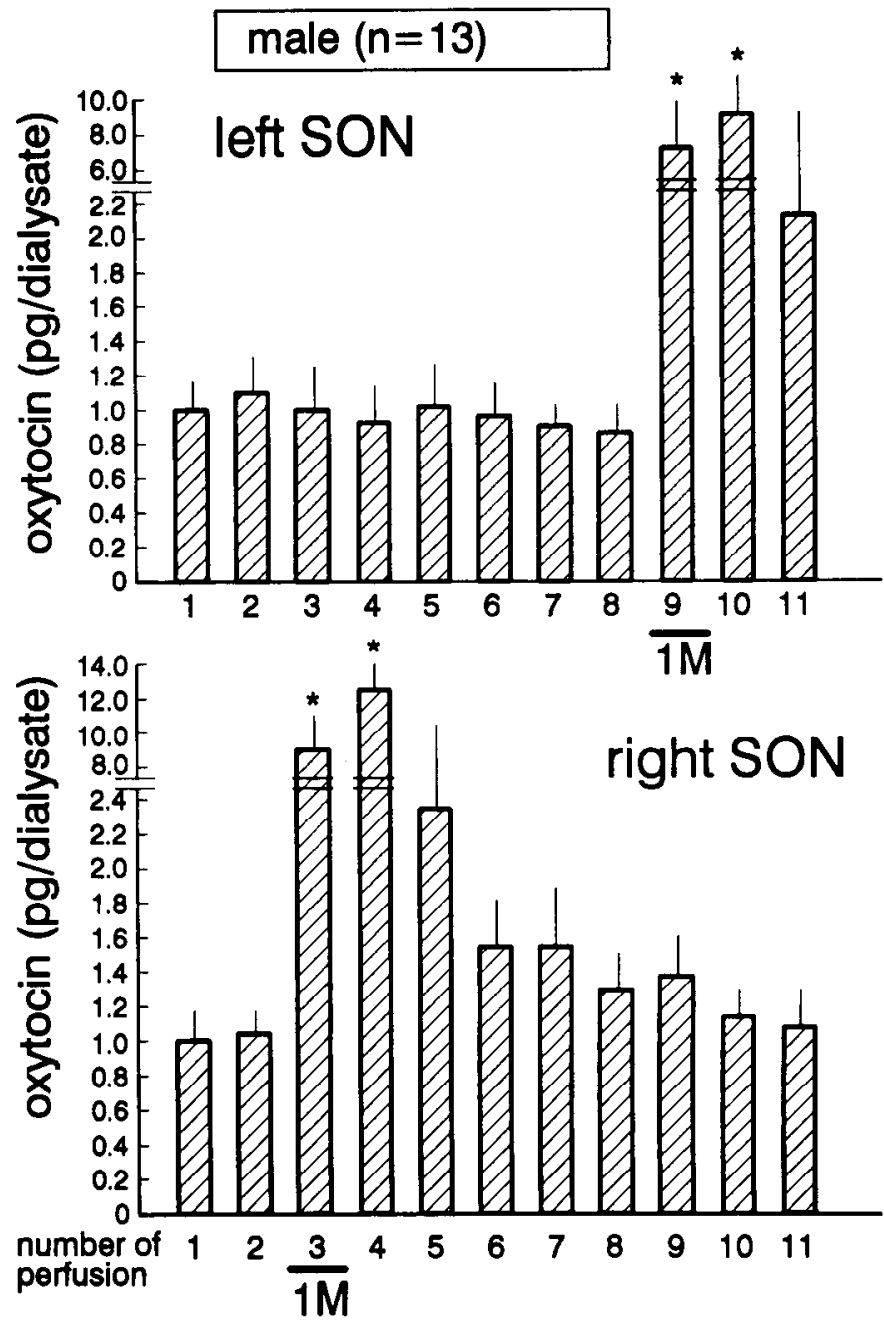

Figure 6. OXT content in 30 min dialysates sampled simultaneously from the left and right SON of male urethane-anesthetized rats. Hypertonic aCSF (with $1 \mathrm{M} \mathrm{NaCl}$ ) was perfused through the right and left probe during the third and ninth perfusion period, respectively. Values are the mean \pm SEM. One-way ANOVA for repeated measurements: $p<0.001$ (both left and right SON) followed by Dunnett's $t$ test, *, $p$ $<0.05$ versus prestimulation.

1987; Higuchi et al., 1988; Koehler et al., 1993), restraint stress (Carter and Lightman, 1987; Higuchi et al., 1988), and injection of CRH (Patel et al., 1991) or CCK (Higuchi et al., 1991) has been described in lactating rats. Various explanations are given for these physiological differences, including reduced stores within the neural lobe (Higuchi et al., 1991), alteration in afferent pathways activating magnocellular neurons, and reduced excitability of OXT neurons themselves (Wakerley and Negoro, 1990). Although electrophysiological evidence is lacking, our results, which demonstrate a reduced release of OXT not only into blood but also within the SON during systemic osmotic stimulation, indicate a reduced excitation of OXT neurons. The precise mechanism for this reduced activation, however, is not known. It is also acknowledged that the stimulation resulting from intraperitoneal hypertonic saline (even in anesthetized animals) may represent a stimulus more complex than that of a strictly osmotic stimulation (Beagley and Hatton, 1993); the contribution of these other, largely undefined factors to the SON responses is still unknown.

Recently, Koehler et al. (1993) reported a blunted peripheral 
Table 1. Vasopression (AVP) content in microdialysates sampled simultaneously from the left and right supraoptic nucleus (SON) of urethane-anesthetized, lactating rats

\begin{tabular}{llllll} 
& Perfusion & & & \\
\cline { 2 - 6 } Dialysate & 1 & 2 & 3 & 4 & 5 \\
\hline Left SON & $1.23 \pm 0.31$ & $1.26 \pm 0.34$ & $1.67 \pm 0.60$ & $7.54 \pm 3.45^{*}$ & $1.17 \pm 0.28$ \\
Right SON & $1.10 \pm 0.26$ & $1.00 \pm 0.21$ & $0.89 \pm 0.13$ & $0.86 \pm 0.13$ & $0.87 \pm 0.10$
\end{tabular}

Five consecutive 30 min microdialysis perfusions were perfomed before ( 1 and 2 ), during (3), and after ( 4 and 5) direct osmotic stimulation of the left SON using hypertonic (with $1 \mathrm{M} \mathrm{NaCl}$ ) instead of isotonic aCSF $(n=6)$. There is no increase of local AVP in the contralateral (right) SON in response to this stimulation. Data are mean \pm SEM.

$* p<0.05$ versus prestimulation levels.

release not only of OXT, but also of AVP after systemic osmotic stimulation in conscious lactating rats. By performing simultaneous microdialysis within the jugular vein and the SON, we could confirm and extend this finding by demonstrating that in contrast to the release of AVP into blood, there is no change in the stimulated release of AVP within the SON due to lactation. Interestingly, the electrically or $\mathrm{K}^{+}$-stimulated release of AVP from isolated neural lobes of virgin and lactating rats was found to be similar (Higuchi et al., 1991).

There was a difference in the time course of OXT and AVP
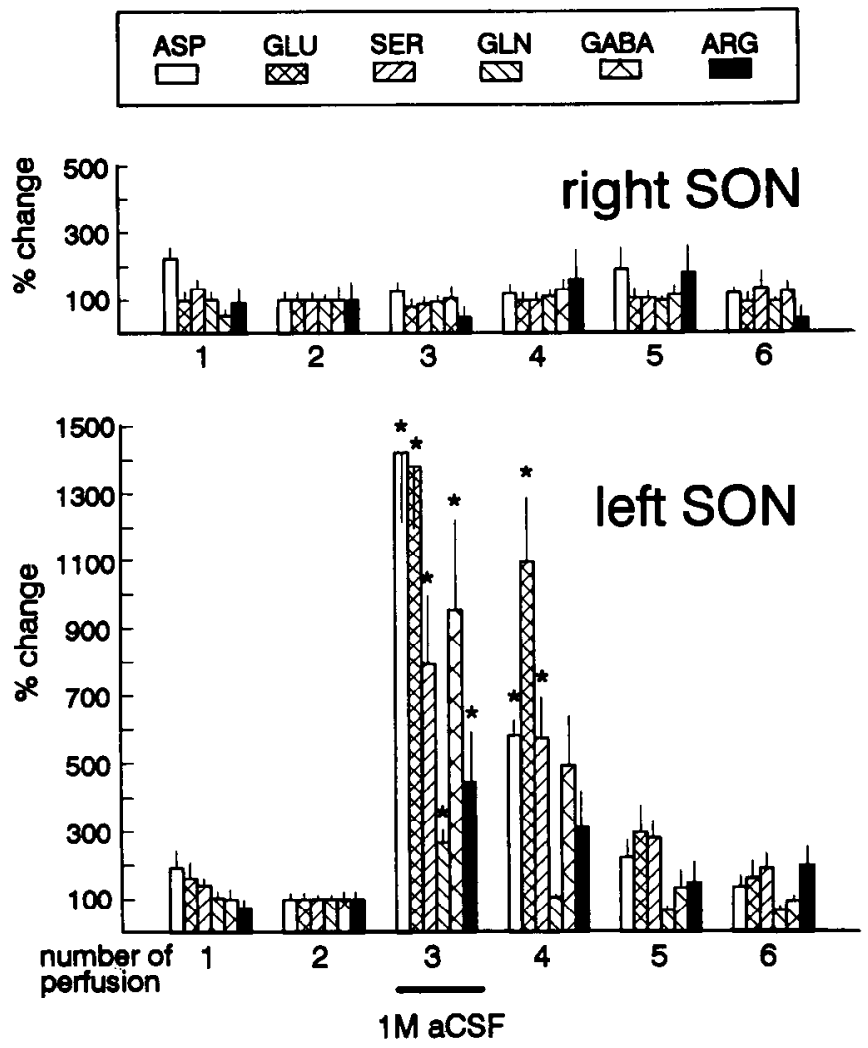

Figure 7. Percent change of anino acid content in six consecutive 15 min dialysates sampled simultaneously from the left and right SON of urethane-anesthetized, lactating rats $(n=6)$. During the third perfusion period, hypertonic aCSF (with $1 \mathrm{M} \mathrm{NaCl}$ ) was perfused through the left dialysis probe. One hundred percent (left SON, in picomoles): $A S P$ (aspartate) $6.33 \pm 1.33, G L U$ (glutamate) $10.5 \pm 2.32, S E R$ (serine) $38.3 \pm 6.67, G L N$ (glutamine) $331 \pm 33.2, G A B A$ ( $\gamma$-aminobutyric acid) $10.8 \pm 2.35, A R G$ (arginine) $20.6 \pm 4.32$. Values are the mean \pm SEM. One-way ANOVA for repeated measurements: $p<0.01$ (arginine), $p<0.001$ (all other amino acids) followed by Bonferroni $t$ test; $*, p<0.05$ versus prestimulation. release into blood (which peaks within the first hour after stimulation) and of release within the SON (which peaks 4-5 hr after i.p. hypertonic saline) in both virgin and lactating animals. However, two questions arise. (1) Is the probe within the SON unable to detect a rise in local peptide concentration as quickly as does the blood probe due to differences in the relative recoveries of the probes (see Material and Methods)? (2) Could a slower clearance rate and/or metabolisin of the SON peptides account for the continued detection of both OXT and AVP over several hours? Both our present data and several earlier studies indicate that the brain dialysis probes can detect, within the first $30 \mathrm{~min}$ sampling period, a significant rise in peptide release caused by local hypertonic saline, as well as release due to suckling or parturition (Neumann et al., 1993a,b). In such cases, the stimulated release returns to basal levels within $30 \mathrm{~min}$ after termination of the stimulus, a time consistent with the rapid disappearance of peptides from a distinct brain site due to processes of diffusion and enzymatic degradation (Burbach, 1985). Therefore, a more likely explanation for the differences in the time course of peptide contents in dialysates after systemic osmotic stimulation is that the probes are detecting independently regulated release patterns of both OXT and AVP into blood and within the SON.

However, different functions of intranuclear OXT during dif-

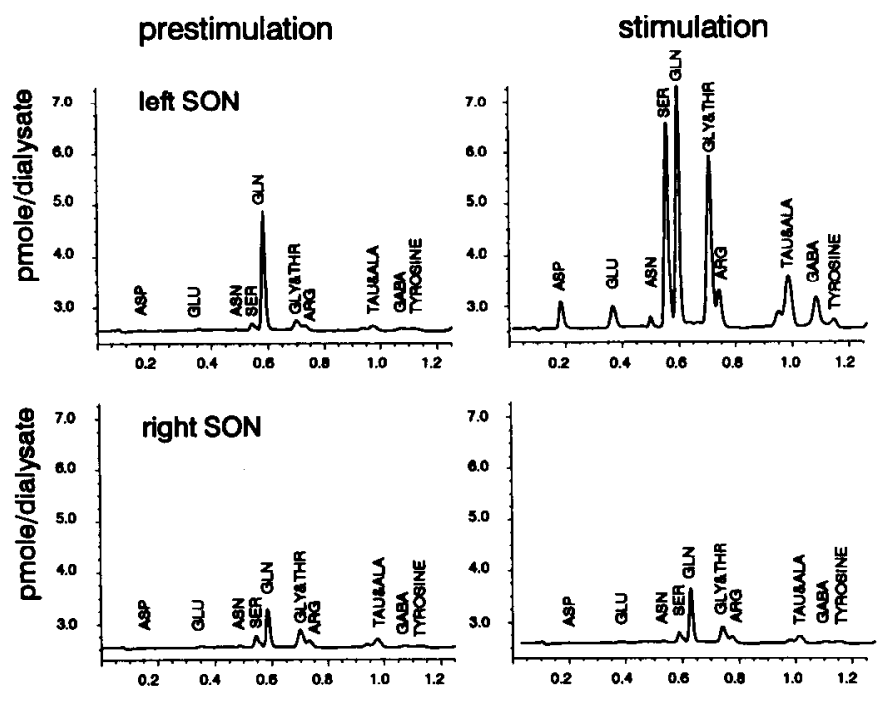

Figure 8. Representative chromatographs indicating amino acid contents in $15 \mathrm{~min}$ dialysates sampled from the left (top) and right SON (bottom) of a urethane-anesthetized, lactating rat before (prestimulation) and during perfusion of the left SON probe with hypertonic aCSF (stimulation). 
ferent physiological challenges may be assumed. During suckling, for example, this intranuclearly released OXT is a prerequisite for the appropriate activation and synchronization of OXT neurons (Lambert et al., 1993) and for OXT release into systemic circulation (Neumann et al., 1994). During systemic osmotic stimulation, peripheral release of OXT (and AVP) peaks before an increase in intranuclear release is detectable. Thus the latter seems not to be essential for efficient peripheral peptide release. Under these particular circumstances, OXT released within the SON might be involved in the structural reorganization of the oxytocinergic system described after osmotic stimulation (Theodosis and Poulain, 1984; Chapman et al., 1986). Interestingly, morphological alterations within the SON are already seen $5 \mathrm{hr}$ after intraperitoneal hypertonic saline (Beagley and Hatton, 1993); that is, at a time when intranuclear OX' and AVP release reach a maximum. The function of intranuclearly released AVP after intraperitoncal hypertonic saline, however, remains unclear, since morphological alterations within the magnocellular nuclei, including withdrawal of glial processes and synaptic rewiring, can be specifically induced by OXT, but not AVP (for review see Theodosis and Poulain, 1993). It would be interesting to know if the delayed increase in intranuclear peptide release is associated with mechanisms related to Fos protein synthesis, which reached a maximum $2 \mathrm{hr}$ after systenic usmotic stimulation (Sharp et al., 1991).

\section{Cross talk between the SON in lactating rats}

The osmotic stimulation of the SON via microdialysis used in this study, although being more of a pharmacological than physiological stimulus, was recently demonstrated to be an efficient means to increase local OXT release within the SON, and from the neurohypophysis into blood in male and female rats (Neumann et al., 1993a,b). In addition, in the present study we demonstrate the release of various excitatory and inhibitory amino acids, like glutamate, aspartate, glycine, serine, and GABA, within the SON in lactating animals in response to local osmotic stimulation, thus confirming recent results in male rats (Horn et al., 1992). The possibility, however, that the hypertonic medium itself, perfused within the ipsilateral SON, or OXT, released within the same SON as a consequence of local osmotic stimulation, may diffuse through the brain tissue thus reaching the contralateral SON has to be excluded. (1) In animals in which the ipsilateral dialysis probe was located outside (even medial to) the SON ( $n=2$; data not shown; see Fig. 1), no increase in contralateral release of OXT was found, which argues against diffusion of the hypertonic medium. (2) The same hypertonic stimulus which caused contralateral release of OXT was ineffective in releasing contralateral AVP or amino acids, again making diffusion of the hypertonic saline to the contralateral side unlikely. (3) Microdialysates sampled outside the contralateral SON of two lactating and two virgin female rats showed no increase in OXT content during or after osmotic stimulation of the SON, which excludes diffusion of OXT to the contralateral site of the brain. (4) The strongest argument, however, is the finding that significant cross talk was found in lactating but not virgin female and male animals, indicating a specific phenom enon related to lactation rather than a nonspecific effect due to diffusion.

Notwithstanding the reduced responses of the oxytocinergic system during lactation to a variety of stimuli, there appear to be compensatory functional adaptations at the level of the OXT neurons to specifically support lactation. OXT cells accelerate their firing rate by $20-40$-fold just before a milk ejection reflex occurs (Wakerley and Lincoln, 1973). Furthermore, the periodic discharge of OXT neurons which is necessary for pulsatile release of efficient amounts of OXT into blood (Higuchi et al., 1986) is highly synchronized within and among different magnocellular nuclei (Belin et al., 1984; Belin and Moos, 1986). OXT released within the SON and the paraventricular nucleus during suckling (Neumann et al., 1993a) plays an important role in the further (positive feedback) activation of local OXT neurons (Neumann et al., 1994). Furthermore, the integrity of strong intra- as well as internuclear connections seems to depend upon local, intranuclear release of OXT (Moos and Richard, 1989; Lambert et al., 1993). These are relatively rapid effects which may have little relationship to the longer time course of the contralateral release described here. However, morphological reorganization of the magnocellular nuclei, especially the SON, which include retraction of glial processes and as a consequence neuronal juxtaposition, as well as synaptic remodeling or electrical coupling (for review see Theodosis and Poulain, 1993), may be influenced in part by contralateral release of OXTO.

The pathways between the left and right SON supporting such functional interactions in lactating rats remain to be identified. Electrophysiological studies demonstrated that these internuclear connections are indirect (Takano et al., 1990). Therefore, we can exclude the possibility that OXT release within the contralateral SON is from OXT terminals originating within the stimulated SON. However, there are bidirectional connections between the SON and the ipsilateral paraventricular nucleus as well as between the paired paraventricular nuclei (Silverman et al., 1981; Saphier and Feldman, 1985, 1987; Moos and Richard, 1989). Cells from the SON that send efferents to the ipsilateral paraventricular nucleus are concentrated in the anterior dorsal aspect, a region where OXT neurons dominate (Sokol et al., 1976). The existence of these pathways, however, is controversial and not confirmed by anterograde transport experiments (Silverman et al., 1981; Tribollet and Dreifuss, 1981; for review see Hatton, 1990). Decussating fibers or interactions between axons/axon collaterals in the stalk or dendrites originating from different nuclei have been proposed as possible ways of communication between the left and right SON (Wakerley and Ingram, 1993). Although the neurochemical characterization remains to be demonstrated, such indirect pathways could be involved in the activation of contralateral OXT neurons. However, we have to take into account the size of the microdialysis membrane (see Fig. 1) and the fact that the direct osmotic stimulation via the microdialysis probe is not limited to the SON only, making it possible that regions dorsal to the nucleus-for example, the perinuclear zone, which has been shown to project into both the SON and paraventricular nucleus (for review see Hatton, 1990)-are stimulated as well.

In most studies, functional relationship and synchronization among OXT neurons are discussed only in relation to lactation. Since also in virgin female, but not in male, rats there was a slight increase in OXT release within the contralateral SON, which, however, did not reach statistical significance, we hypothesize the existence of established pathways connecting the SONs in female rats which become further activated during lactation. This is supported by our recent finding of an increased glucose metabolism in the contralateral SON of both virgin and lactating rats after direct osmotic stimulation of one SON (Summy-Long et al., 1994).

Other neurotransmitter candidates might be involved in the 
cross talk among the SONs and the stimulation of contralateral OXT neurons; glutamate and GABAergic neurons, for example, innervate magnocellular neurons (Theodosis et al., 1986b; Decavel and van den Pol, 1990; van den Pol et al., 1990; Crowley and Armstrong, 1992). Interestingly, in dialysates sampled from the contralateral SON of lactating rats neither AVP nor any of the amino acids measured showed an increased content during osmotic stimulation of one SON. It is thus tempting to speculate that there is a specific activation of contralateral oxytocinergic neurons during stimulation of one SON.

Our results do not rule out, however, the participation of amino acid transmitters in the excitation of contralateral OXT neurons. In lactating rats $\mathrm{OXT}$ neurons might be more sensitive to any inputs arriving from the contralateral SON. Thus, the activation of only a few projections to the contralateral SON, which might be undetectable by the means used, might be efficient enough to stimulate oxytocinergic neurons and OXT release within the SON. It is also possible that excitation of the contralateral nucleus may be caused by a fall in levels of an inhibitory amino acid such as GABA.

The present data suggest that functional adaptations of the oxytocinergic system during lactation may compensate for the reduced release of the neuropeptide within the SON and into blood in response to non-lactation-related stimuli. One of those alterations might be the activation of (still unidentified) indirect pathways which connect the left and right SON to coordinate OXT release in response to physiological demands.

\section{References}

Beagley GH, Hatton GI (1993) Systemic signals contribute to induced morphological changes in the hypothalamo-neurohypophysial system. Brain Res Bull 33:211-218.

Belin V, Moos F (1986) Paired recordings from supraoptic and paraventricular oxytocin cells in suckled rats: recruitment and synchronization. J Physiol (Lond) 377:369-390.

Belin V, Moos F, Richard PH (1984) Synchronization of oxytocin cells in the hypothalamic supraoptic and paraventricular nuclei in suckled rats: direct proof with paired extracellular recordings. Exp Brain Res 57:201-203.

Burbach JPH (1985) Proteolytic conversion of oxytocin, vasopressin, and related peptides in the brain. In: Current topics in neuroendocrinology, Vol 6, Ncurobiology of oxytocin (Ganten D, Pfaff D, eds), pp 55-90. Berlin: Springer.

Carter DA, Lightman SL (1987) Oxytocin responses to stress in lactating and hyperprolactinaemic rats. Neuroendocrinology 46:532537.

Chapman DB, Theodosis DT, Montagnese C, Poulain DA, Morris JF (1986) Osmotic stimulation causes structural plasticity of neuroneglia relationships of the oxytocin but not vasopressin secreting neurones in the hypothalamic supraoptic nucleus. Neuroscience 17:679 686.

Crowley WR, Armstrong WE (1992) Neurochemical regulation of oxytocin secretion in lactation. Endocr Rev 13:33-65.

Decavel C, van den Pol AN (1990) GABA: a dominant neurotransmitter in the hypothalamus. J Comp Neurol 302:1019-1037.

Hartman RD, Rosella-Dampman LM, Summy-Long JY (1987) Endogenous opioid peptides inhibit oxytocin release in the lactating rat after dehydration and urethane. Endocrinology 121:536-543.

Hatton GI (1990) Emerging concepts of structure-function dynamics in adult brain: the hypothalamo-neurohypophysial system. Neurobiology 34:437-504.

Higuchi T, Tadokoro Y, Honda K, Negoro H (1986) Detailed analysis of blood oxytocin levels during suckling and parturition in the rat. $\mathrm{J}$ Neuroendocrinol 1 10:251-256.

Higuchi T, Honda K, Takano S, Negoro H (1988) Reduced oxytocin response to osmotic stimulus and immobilization stress in lactating rats. J Fndocrinol 116:225-230.

Higuchi T, Bicknell RJ, Leng G (1991) Reduced oxytocin release from the neural lobe of lactating rats is associated with reduced pituitary content and does not reflect reduced excitability of oxytocin neurons. J Neuroendocrinol 3:297-302.

Horn T, Bauce L, Landgraf R, Pittman QJ (1992) Microdialysis with hypertonic $\mathrm{NaCl}$ causes central release of amino acids and dopamine. In: Proceedings of the 18th Meeting of the Society of Neuroscience, Anaheim, 421.10

Koehler E, McLemore GL, Tang W, Summy-Long JY (1993) Osmoregulation of the magnocellular system during pregnancy and lactation. Am .I Physiol 264:R555-R560.

Lambert RC, Moos FC, Richard Ph (1993) Action of endogenous oxytocin within the paraventricular or supraoptic nuclei: a powerful link in the regulation of the bursting pattern of oxytocin neurons during the milk-ejection reflex in rats. Neuroscience 57:1027-1038.

Moos F, Richard Ph (1989) Paraventricular and supraoptic bursting oxytocin cells in the rat are locally regulated by oxytocin and functionally related. J Physiol (Lond) 408:1-18.

Moos F, Poulain DA, Rodriguez F, Guerne Y, Vincent JD, Richard P (1989) Release of oxytocin within the supraoptic nucleus during the milk ejection reflex in rats. Exp Brain Res 76:593-602.

Neumann I, Russell IA, I andgraf R (1993a) Oxytocin and vasopressin release within the supraoptic and paraventricular nuclei of pregnant, parturient and lactating rats: a microdialysis study. Neuroscience 53: $65-75$.

Neumann I, Ludwig M, Engelmann M, Pittman QJ, Landgraf R (1993b) Simultaneous microdialysis in blood and brain: oxytocin and vasopressin release during osmotic stimulation and suckling in the rat. Neuroendocrinology 58:637-645.

Neumann I, Koehler E, Landgraf R, Summy-Long JY (1994) Administration of an oxytocin receptor antagonist into the rat supraoptic nucleus attenuates intranuclear and peripheral oxytocin release in conscious lactating rats during suckling. Endocrinology 134:141-149.

Patel H, Chowdry HS, Lightman SL (1991) Lactation abolishes corticotropin-releasing factor-induced oxytocin secretion in the conscious rat. Endocrinology 128:725-727.

Paxinos G, Watson C (1986) The rat brain in stereotaxic coordinates. Sydney: Academic.

Pow DV, Morris JF (1989) Dendrites of hypothalamic magnocellular neurons release neurohypophysial peptides by exocytosis. Neuroscience 32:435-440.

Saphier D, Feldman S (1985) Evidence for neural connections between the paraventricular nucleus and neurons of the supraoptic nucleus in the rat. Exp Neurol 89:289-294.

Saphier D, Feldman S (1987) Electrophysiology of supraoptico-paraventricular nucleus connections in the rat. Exp Brain Res 69:60-66.

Sharp FR, Sagar SM, Hicks K, Lowenstein D, Hisanaga K (1991) Cfos mRNA, Fos, and Fos-related antigen induction by hypertonic saline and stress. J Neurosci 11:2321-2331.

Silverman AJ, Hoffman DL, Zimmerman EA (1981) The descending afferent connections of the paraventricular nucleus of the hypothalamus (PVN). Brain Res Bull 6:47-61.

Sokol HW, Zimmerman EA, Sawyer WH, Robinson AG (1976) The hypothalamo-neurohypophysial system of the rat: localization and quantification of neurophysin by light microscopic immunocytochemistry in normal rat and in Brattleboro rat deficient in vasopressin and a neurophysin. Endocrinology 98:1176-1188.

Summy-Long JY, Neumann I, Terrell ML, Koehler E, Mantz S, Landgraf R, Kadekaro M (1994) Metabolic crosstalk in hypothalamic magnocellular nuclei during osmotic stimulation of one SON. Brain Res Bull 33:645-654.

Takano S, Negoro H, Honda K, Higuchi T (1990) Electrophysiological evidence for neural connections between the supraoptic nuclei. Neurosci Lett 111:122-126.

Theodosis DT (1985) Oxytocin-immunoreactive terminals synapse on oxytocin neurones in the supraoptic nucleus. Nature 313:682-684.

Theodosis DT, Poulain DA (1984) Evidence that oxytocin-secreting neurones are involved in the ultrastructural reorganisation of the rat supraoptic nucleus apparent at lactation. Cell Tissue Res 235:217219.

Theodosis DT, Chapman DB, Montagnese C, Poulain DA, Morris JF (1986a) Structural plasticity in the hypothalamic supraoptic nucleus at lactation affects oxytocin-, but not vasopressin-secreting neurones. Neuroscience 17:661-678.

Theodosis DT, Paut L, Tappaz ML (1986b) Immunocytochemical analysis of the GABAergic innervation of oxytocin- and vasopressin- 
secreting neurons in the rat supraoptic nucleus. Neuroscience 19:207222.

Tribollet E, Dreifuss JJ (1981) Localization of neurones projecting to the hypothalamic paraventricular nucleus area of the rat: a horseradish peroxidase study. Neuroscience 6:1315-1328.

Van den Pol AN, Wuarin JP, Dudek FE (1990) Glutamate, the dominant excitatory transmitter in neuroendocrine regulation. Science 250: 1276-1278.
Wakerley JB, Ingram CD (1993) Synchronization of bursting in hypothalamic oxytocin neurones: possible coordinating mechanisms. News Physiol Sci 8:129-133.

Wakerley JB, Lincoln DW (1973) The inilk-ejection reflex of the rat: a 20 - to 40 -fold acceleration in the firing of paraventricular neurones during oxytocin release. J Endocrinol 57:477-493.

Wakerley JB, Negoro H (1990) Evidence for altered sensitivity of supraoptic neurones to phenylephrine in lactating versus non-lactating rats. Neuroendocrinology 52[Suppl 1]:118. 